\title{
Medidas de gestión y adecuación ambiental de embalses frente a la eutrofia
}

\author{
Antoni Palau Ibars \\ Departamento de Medio Ambiente y Desarrollo Sostenible. Endesa Servicios S.L. Carretera de Tarragona, \\ Km 89,300. 25001-Magraners (Lleida)
}

\section{RESUMEN}

La eutrofización es una alteración prácticamente inherente a la construcción de embalses y a su explotación. Ciertamente, existen diferentes tipos de embalses y no en todos la eutrofia se manifiesta con la misma intensidad. Por la misma razón, no siempre son aplicables con igual éxito las distintas medidas de atenuación del grado de eutrofia que se conocen.

En la ponencia se muestra una revisión cualitativa, pero suficientemente exhaustiva, de las medidas de predicción, prevención y corrección de la eutrofia, asociables a embalses, en sentido amplio.

En cualquier propuesta de control de perturbaciones provocadas por actuaciones humanas en sistemas naturales (como es el caso de la construcción de embalses), el punto de partida es la predicción; es decir, el reconocimiento previo a la actuación, de cual será la respuesta del medio a la perturbación en función de las características de este. Conocida la respuesta más probable, si esta se evalúa como crítica a los efectos de los objetivos de la obra hidráulica proyectada y/o a los costes y funcionalidad (eficiencia) de su explotación, se pueden adoptar una serie de medidas preventivas con el fin de minimizar en lo posible, la eutrofia y/o sus síntomas.

En la fase de explotación de un embalse, se reducen los grados de libertad en cuanto a posibles actuaciones de control y atenuación de la eutrofia y adquiere una importancia decisiva los usos a los cuales se dedica el embalse (abastecimiento urbano, riegos, hidroelectricidad, etc.) a la hora de escoger las opciones de actuación.

Finalmente, la eutrofia es una alteración que no sólo afecta a los embalses, sino también a los tramos de río aguas abajo de ellos de modo que es necesario contemplar también medidas de control en este tipo de ambientes.

Palabras clave: Eutrofización, embalse, medidas de control y gestión.

\begin{abstract}
Eutrophication is a rather inherent ecological disturbance of dam building and reservoir management. Certainly, there are different types of dams and not all of them show the same degree of eutrophication. For the same reason, not all the known eutrophy control measures can be applied with the same success. In this paper a qualitative but rather exhaustive revision is presented, about eutrophy's prediction, prevention, management and correction measures that can be related to dams in a general sense. As with any proposal for control of human disturbances in natural systems (such as the case of dam building), the starting point is prediction; that is, the acknowledgement before action is taken, about what will be the response of the environment to that perturbation according to its' characteristics. Once the most likely response in terms of potential eutrophication is known, and if this response is evaluated as a critique to the effects of the projected hydraulic construction objectives, and/or to the costs and functionality (efficiency) of it's operation, then a number of preventive measures can be adopted to minimize, as much as possible, eutrophy and/or its'symptoms. During a dam's operation phase, the degrees of freedom for possible eutrophy control and mitigation actions are reduced with respect to other phases, and the uses to which the dam is oriented (urban water provision, irrigation, hydroelectricity generation, etc.) become critical when is time to select eutrophy's control options. Finally, eutrophication is a change that not only affects reservoirs, but also sections of the rivers downstream the dam, so it is also necessary to keep in mind the control measures for this type of environment.
\end{abstract}

Keywords: Eutrophication, reservoir, control and management measures. 


\section{INTRODUCCIÓN}

La eutrofización denominada "cultural" es percibida desde hace años como una alteración preocupante de la calidad de las aguas continentales (Margalef, 1976; Lee et al., 1980) y de amplia distribución en los ecosistemas acuáticos. En algunos lugares, no obstante, la eutrofización no es vivida como una perturbación de los ecosistemas acuáticos sino como una forma de cultivo acuático, fuente de alimento, que hay que potenciar (Ryding \& Rast, 1993). Por otro lado, la eutrofia como proceso y sus consecuencias, bien pudo jugar un papel clave hace unos 2.000 millones de años, en la transformación de la atmósfera de la tierra hacia las condiciones oxidantes actuales.

En el caso de los embalses es una alteración prácticamente inherente a su construcción y a su explotación, especialmente en zonas semiáridas, bien soleadas, con un régimen hidrológico marcadamente estacional y redes hidrográficas densamente ocupadas por población o usos agropecuarios. La mayor susceptibilidad de los embalses a la eutrofia, con relación a los lagos, se explica por la carga de materia orgánica que los primeros deben procesar de golpe en su inicio y por la alta relación entre las superficies de cuenca y de lámina de agua, que favorece el mantenimiento de unas mayores aportaciones relativas de nutrientes por unidad de superficie.

Este artículo no es ni pretende ser una revisión bibliográfica sobre las medidas de gestión y adecuación ambiental de los embalses frente a la eutrofia. Se trata de una recopilación cualitativa de propuestas fundamentadas en actuaciones, técnicas y procedimientos, que se sabe, en algunos casos por experiencia propia, pueden dar buenos resultados en el control de la eutrofia de los embalses, tanto desde un punto de vista predictivo, como preventivo o corrector.

Son en general propuestas bien conocidas desde hace años, recopiladas de diferentes fuentes, que se consideran absolutamente vigentes en la actualidad e incluso, algunas de ellas, insuficientemente documentadas, contrastadas y sancionadas por la práctica.
Un par de aspectos importantes son los siguientes. En primer lugar hay tener en cuenta que existe una amplia tipología de embalses y por tanto de condiciones y condicionantes para la manifestación de la eutrofia. Por esta razón, no todas las propuestas de control de la eutrofia son aplicables a todos los embalses y, en el caso de las que presentan un más amplio espectro de aplicación, no necesariamente han de alcanzar la misma eficiencia en cualquier embalse donde se apliquen.

En segundo lugar, si bien la eutrofización es un concepto originariamente acuñado para ecosistemas leníticos, afecta también y de forma muy significativa a los tramos de río regulados por embalses eutróficos, de modo que las actuaciones de prevención y corrección deben contemplar también esa parte del ecosistema fluvial.

\section{EL CONTROL DE LA EUTROFIA EN EMBALSES}

Las actuaciones de control de la eutrofia en embalses deben tratarse como cualquier otro aspecto técnico constructivo relevante y como tal deben empezar a considerarse en la propia concepción del proyecto de construcción de la presa que formará el embalse, o en su defecto, en el estudio de impacto ambiental que le acompaña. A este nivel, la primera cuestión a plantearse es la vocación trófica que tendrá el futuro embalse (Fig. 1), de acuerdo con la información disponible sobre el lugar donde se pretende construir y las características básicas del tipo de obra hidráulica que se proyecta.

Esta fase de predicción del grado de eutrofia que tendrá el embalse, puede tener un margen de error apreciable, pero debe permitir en cualquier caso precisar la necesidad o no de proyectar y planificar medidas de atenuación de la eutrofia. En esta fase es donde tiene mayor sentido la detección de las causas y/o los orígenes de la posible situación de eutrofia, al objeto de evaluar las opciones de actuación sobre ellos.

El diseño y la consideración a nivel de proyecto (o de su correspondiente estudio de 


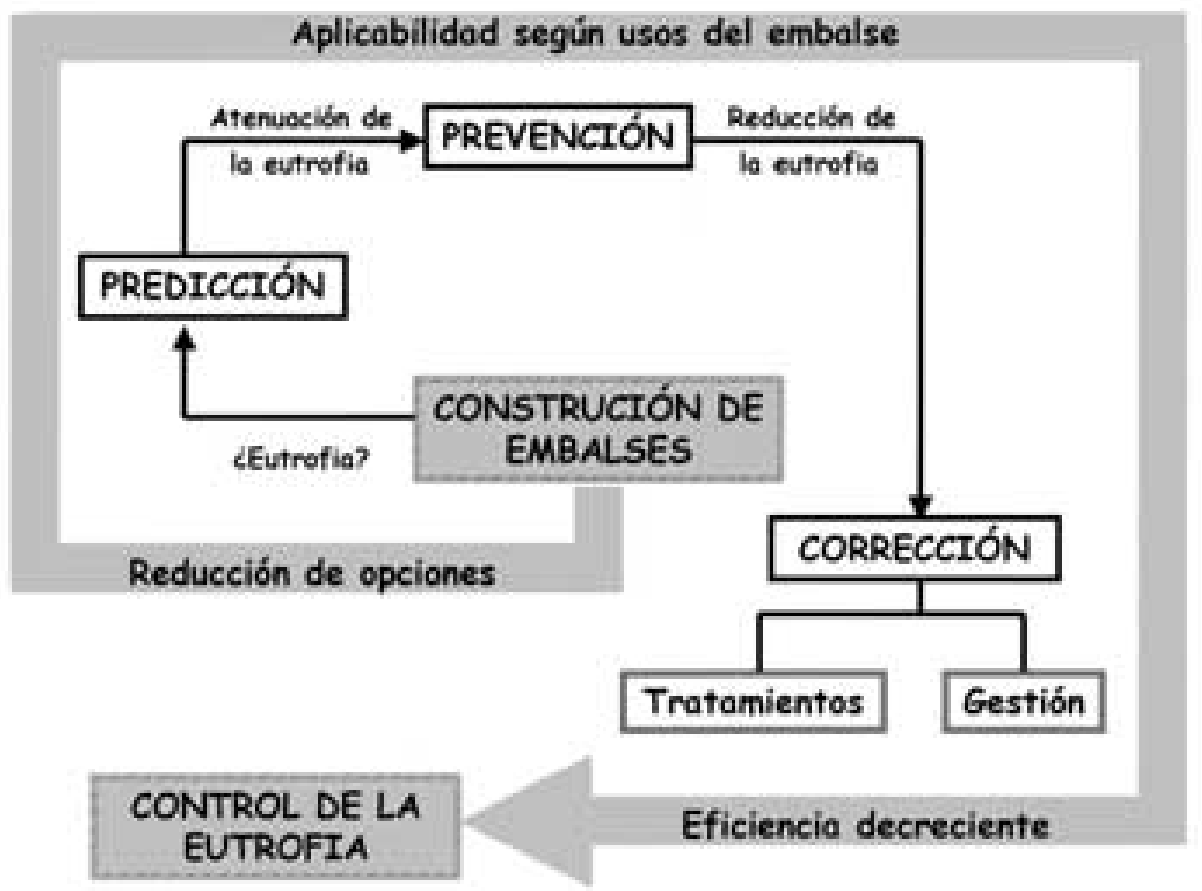

Figura 1. Esquema general del enfoque que recibe el control de la eutrofia en el presente artículo. General diagram of the eutrophication control approach in this paper.

impacto ambiental), de las posibles medidas de atenuación de la eutrofia, constituye la fase de prevención, que podrá incluir tanto obras e infraestructuras, como actuaciones de gestión ya programadas, encaminadas a evitar, en la medida de lo posible, la eutrofización de la masa de agua embalsada.

Finalmente, si a pesar de todo se llega (o existe) un grado de eutrofia inaceptable, bien sea desde un punto de vista ecológico, socioeconómico o de explotación de la obra hidráulica, no quedan más opciones que la adopción de medidas correctoras que, se aplicarán fundamentalmente sobre los síntomas de eutrofia, en forma de tratamientos y/o gestión del volumen de agua embalsada.

A lo largo de estas tres fases (predicción, prevención y corrección) desde la concepción de un proyecto de embalse hasta la consecución de un nivel aceptable de eutrofización, se van perdiendo grados de libertad a nivel de posibilidades de actuación, en el sentido que las opciones de aplicación de medidas (obras, actuaciones, etc.) se van reduciendo o encareciendo, desde la fase de proyecto hasta la de explotación del embalse; es decir la falta de previsión en las consideraciones iniciales del proyecto de embalse, redunda en posibles limitaciones del uso del agua o en unos costes de control de la eutrofia crecientes, en la fase de explotación, lo que en definitiva puede interpretarse como una pérdida de la eficiencia o rendimiento del proyecto de embalse y el uso del agua, en la medida que la relación coste/beneficio aumenta.

\section{Predicción de la vocación trófica}

La eutrofia no se manifiesta por igual en cualquier ecosistema acuático, siendo los más afectados los de tipo lenítico (lagos y embalses) y aún dentro de estos, una misma aportación de fósforo 
Tabla 1. Información básica en la predicción de la vocación trófica de un futuro embalse y de sus consecuencias río abajo. Basic information for the prediction of possible trophic states of a future reservoir and its' consequences downstream.

\section{FUENTES DE INFORMACIÓN}

Localización geográfica del futuro embalse

\section{ASPECTOS BÁSICOS A CONSIDERAR}

Características del anteproyecto

de embalse

\author{
Climatología \\ Hidrología \\ Hidrogeología \\ Características físico-químicas de las aguas \\ Usos y estado de conservación de la cuenca \\ Usos del futuro vaso de embalse \\ Morfología del cauce y riberas en el tramo regulado \\ Usos y estado de conservación de la ribera en el tramo regulado
}

Morfología del futuro vaso de embalse

Usos y manejo del agua embalsada

Profundidad y características de las tomas de agua en la presa

Caudales de mantenimiento en el tramo regulado puede tener efectos muy distintos según algunas características del ecosistema acuático receptor.

Toda predicción supone deducir la respuesta de un proceso o sistema concretos, frente a unas determinadas condiciones y por tanto en el caso de la eutrofización de embalses, hay que partir del conocimiento del tipo de embalse que se proyecta y de las características del medio donde se pretende construir. La tabla 1 muestra la información básica necesaria para la predicción.

A partir de la localización geográfica del lugar donde se proyecta el embalse y de su anteproyecto constructivo, no resulta demasiado complicado obtener información sobre los aspectos referidos en la tabla 1. De ellos puede deducirse, o al menos estimarse, el origen y la carga de nutrientes y/o de materia orgánica que recibirá el embalse, la dureza del agua y su contenido habitual en oxígeno disuelto o bien el tiempo medio de residencia del agua embalsada y su circulación por el vaso de embalse, así como el tipo de ciclo térmico anual del embalse y su estabilidad térmica, relacionándolo todo ello con la disponibilidad y el comportamiento de nutrientes. También puede obtenerse una serie de índices referidos a las aptitudes morfológicas del futuro vaso de embalse (Wetzel, 1981) de cara a la eutrofización que, aún no siendo definitivos, complementan el análisis de la vocación trófica de un embalse. Conocidos estos aspectos, la predicción de la eutrofia, en la mayoría de casos, pasa por la cuantificación de las cargas de fósforo que recibirá el embalse y la modelización de sus consecuencias mediante alguno de los modelos, de diferente complejidad y precisión, que existen para la determinación de la vocación trófica del futuro embalse (Ryding \& Rast, 1993).

Para el reconocimiento de los posibles efectos del estado trófico del embalse en el río aguas abajo, interesa conocer la profundidad de la toma (o tomas) de agua desde el embalse, así como las características de la incorporación de esas aguas al río. Resulta también esencial saber el régimen de caudales de mantenimiento y de explotación del embalse, así como las características morfológicas, hidráulicas y limnológicas del cauce y las riberas que afectarán a su capacidad de procesado de nutrientes y, en definitiva, a la intensidad de la manifestación de la posible eutrofia.

La estimación de las cargas de nutrientes y materia orgánica que recibe el cauce principal y los tributarios río abajo, permite reconocer posibles efectos sinérgicos o acumulativos, en especial en embalses que bien sea por capacidad de regulación o de derivación de aguas, reducen drásticamente los caudales circulantes río abajo. 


\section{Prevención de la eutrofia}

El principal resultado de la predicción sobre la vocación trófica de un futuro embalse, es el reconocimiento de una parte de los posibles efectos ambientales del embalse proyectado sobre el ecosistema fluvial y la identificación de las limitaciones que la eutrofia puede suponer para los usos y objetivos de explotación del embalse. De todo ello, se podrán deducir las necesidades (y posibilidades) de actuación en la reducción de las cargas de nutrientes o en la atenuación de los síntomas de la posible eutrofización, que haya que incorporar al proyecto constructivo, con carácter preventivo. La Tabla 2 recoge las consideraciones más habituales en la prevención de la eutrofia, susceptibles de tenerse en cuenta en nuevos embalses y algunas de ellas también como medidas de corrección del grado de eutrofia en embalses ya existentes.

Las actuaciones preventivas, para ser eficaces, deben quedar recogidas en el propio proyecto constructivo o en el preceptivo estudio de impacto ambiental que le acompaña. Es importante destacar que todas estas actuaciones, a diferencia de las indicadas en la fase de predicción, suponen ya unos costes económicos importantes y unos compromisos (decisiones) bastante irreversibles.

Con relación al diseño de las obras, equipos e infraestructuras (tabla 2) una primera consideración debe ser siempre el análisis de la reducción

Tabla 2. Niveles de actuación y posibles aspectos a considerar en cada uno de ellos, con relación a la prevención de la eutrofización en futuros embalses y la reducción de sus efectos negativos en el río aguas abajo. Levels of intervention and possible issues to be incorporated to each one of them, in relation to eutrophication prevention the eutrophication of a new reservoir and to reduce its negative consequences downstream the dam.

\section{NIVEL DE ACTUACIÓN}

Proyecto técnico o estudio de impacto ambiental. Aspectos referidos a obras, equipos e infraestructuras

Proyecto técnico o estudio de impacto ambiental. Aspectos referidos al tratamiento previo del futuro vaso de embalse

Proyecto técnico o estudio de impacto ambiental. Aspectos referidos a los usos del embalse

Proyecto técnico o estudio de impacto ambiental. Planificación del primer llenado del embalse

Proyecto técnico o estudio de impacto ambiental. Gestión del tramo de río regulado

\section{POSIBLES ASPECTOS A CONSIDERAR}

- Depuración de aguas residuales en la cuenca tributaria

- Restauración hidrológico-forestal de la cuenca

- Desviación ("by-pass") de tributarios

- Características y ubicación (localización en profundidad) de las tomas de agua

- Dimensionado del vaso de embalse

- Construcción de presas de cola

- Reducción (extracción) de la carga de materia orgánica oxidable

- Aprovechamiento de recursos inundables (suelos, vegetación, madera, áridos)

- Usos y tiempos de residencia del agua

- Ordenación (limitación) de usos y actividades en el embalse

- Época del año para el inicio del llenado del embalse

- Curva (velocidad) de llenado de embalse

- Plan de extracción selectiva de agua a diferentes profundidades según la época del año

- Vaciados periódicos totales o parciales del embalse

- Régimen de caudales de mantenimiento

- Restauración y/o consolidación de la vegetación de ribera

- Capacidad de aireación del cauce

- Características y ubicación (localización en profundidad) de las tomas de agua 
en origen del exceso de nutrientes que vaya a recibir el embalse. Así, en función de la importancia socioeconómica del embalse, sería admisible plantearse actuaciones de saneamiento de aguas residuales directas y difusas, así como la restauración hidrológico-forestal a nivel de la cuenca tributaria. Una opción hasta cierto punto alternativa puede ser la construcción de una presa de cola, que además de poder contribuir a la retención (y extracción de nutrientes), a menudo puede convertirse en una infraestructura social y económicamente dinamizadora de la zona, si se diseña con capacidad para acoger actividades lúdicas y recreativas. Por otro lado, no es infrecuente que estas zonas represadas en las colas de embalses, acaben convirtiéndose en zonas húmedas con cierto valor de conservación. En el mismo sentido, si las condiciones del entorno del embalse y sus dimensiones lo permiten, puede plantearse la desviación parcial (o temporal) del tributario responsable de la eutrofización, hacia zonas húmedas de alrededor o río abajo, si bien hay que tener en cuenta que con ello se modifica la tasa de renovación del agua embalsada, la estructura de la comunidad algal y las relaciones agua-sedimentos (Moss et al., 1989).

El dimensionado del vaso de embalse suele admitir pocas modificaciones, dado que está acotado por las características topográficas de la cerrada donde se proyecta la presa y por los objetivos del embalse, que corren el riesgo de perder sentido si se altera significativamente el volumen embalsable. En cambio, la ubicación y el diseño de las tomas de agua, desde las superficiales hasta los desagües de fondo, si admite cambios que no sólo no tienen porque penalizar el aprovechamiento del embalse, sino que pueden mejorarlo al aumentar las garantías de explotación; así, en la utilización de uno u otro tipo de válvula o compuerta en una toma, o también en su profundidad, puede estar la diferencia en suministrar río abajo unas aguas con poco o nada de oxígeno disuelto durante la estratificación estival o bien prácticamente saturadas al $100 \%$ durante cualquier época del año.

Sobre el tratamiento previo del futuro vaso de embalse, son bien conocidas las propuestas de retirada de la vegetación como medida de reducción de la carga orgánica oxidable bajo el agua. La eficacia de esta medida no siempre ha quedado demostrada, dado que una fracción importante de la materia orgánica oxidable se encuentra en los horizontes superiores del suelo, componente este mucho más difícil de retirar, a pesar de ser, ecológica y económicamente, bastante más valioso que la cubierta vegetal. En cualquier caso, cualquier aprovechamiento que pueda hacerse de los recursos situados en la zona inundable por el embalse (madera, suelos, áridos, etc.) es, por principio, recomendable.

En cuanto a la prevención aplicada a los usos del agua y del embalse, dado que como ya se ha indicado el dimensionado de su volumen no es fácilmente modificable y la aportación de los tributarios tampoco (salvo si son ríos regulados), resulta que las posibilidades de gestión de los tiempos de residencia medios del total de agua embalsada, aspecto realmente clave en la prevención y la contención de la eutrofia (Straskraba, 1999), son limitados. Sin embargo, si se dispone de un sistema de tomas de agua a distintas profundidades suficientemente versátil (Cassidy, 1989), puede llevarse a cabo una actuación eficaz en el control de la producción primaria y por tanto de la manifestación de la eutrofia, mediante una gestión selectiva de la tasa de renovación del agua a diferentes profundidades y épocas del año (Toja, 1982; Palau, 1991).

Las propuestas de ordenación de usos y actividades son una opción con signo preventivo, pero de interés también en casos de embalses en explotación sujetos a aprovechamientos diversos, tanto de tipo hidráulico (riegos, producción hidroeléctrica, abastecimientos) como de tipo recreativo (navegación, pesca, etc.). En algunos casos, los resultados en materia de control y prevención de la eutrofia pueden ser apreciables a partir de medidas tan simples y aparentemente tan indirectas como la gestión de la pesca deportiva, por ejemplo controlando los efectos de la resuspensión de nutrientes (bioturbidez) por un exceso de ejemplares de gran tamaño de determinadas especies de peces de hábitos bentónicos detritívoros. En el mismo sentido, la 
ordenación de otros usos frecuentes en embalses, como el baño y la navegación recreativa, además de lógicamente el control de vertidos directos o difusos al embalse mediante una planificación de usos y actividades en sus orillas y márgenes (Wilson \& O'Sullivan, 1993) también pueden contribuir significativamente en la prevención y mitigación de la eutrofia.

Pero independientemente de los resultados que se consigan con medidas preventivas del tipo de las indicadas, no hay que olvidar que la eutrofia es, en la práctica, una condición inherente a cualquier nuevo embalse, de modo que siempre habrá que contar, con un proceso de eutrofia inicial más o menos intenso, asociado al primer llenado del embalse.

Una forma de incidir en una reducción de la intensidad y la duración de este proceso de maduración de un nuevo embalse es mediante una planificación del primer llenado del embalse, que debe tener en consideración al menos los aspectos relacionados al respecto en la tabla 2 . Si bien no siempre es posible aplicar una programación de este tipo, en general es mejor proceder al primer llenado con aguas frías (más densas y oxigenadas) e ir llenando el embalse progresivamente en varios años, con unos tiempos de residencia del agua lo más bajos posible al inicio, gestionados mediante vaciados parciales (o totales, según el volumen de embalse y la aportación), preferentemente con extracción selectiva del agua a diferentes profundidades según la época del año. Los costes añadidos que puede suponer la aplicación de esta medida, se ven compensados en la mejor calidad del agua embalsada, lo que reduce la afectación ambiental del embalse en el río y no limita ni condiciona su explotación por tanto tiempo como el que se requiere sin una gestión del primer llenado.

Río abajo, las medidas preventivas frente a la eutrofización de un embalse tienen como objetivo común el favorecer la mezcla y la aireación del agua, junto con el mantener (o restablecer) el control trófico que la vegetación de ribera realiza sobre el cauce. Así, se trata de implantar un régimen de caudales de mantenimiento que contemple, no sólo un caudal mínimo o "ecoló- gico" sino una distribución temporal del caudal acorde con el régimen hidrológico natural, incluyendo crecidas controladas (caudales generadores o renovadores) y otras consideraciones (Palau, 1994). Estos caudales de mantenimiento deben permitir una adecuada aireación del cauce y una limitada proporción de ambientes limnófilos, más propensos a permitir las formaciones de macrófitos y con ello a reconcentrar en un tramo habitualmente demasiado corto, la mayor parte del reciclado de nutrientes procedentes del embalse. Cabe indicar que en algunos casos sería planteable una situación totalmente contraria a la descrita; es decir dedicada a favorecer el estancamiento del agua en las inmediaciones del pie de presa para que la producción primaria del cauce retenga la mayor cantidad posible de nutrientes, evitando su dispersión aguas abajo.

Por otro lado, la restauración y/o consolidación de la vegetación de ribera, en la medida que ejerce un control sobre la temperatura del agua y la disponibilidad de luz y nutrientes para los productores primarios acuáticos, es también de interés si la opción escogida es la del procesado dosificado de los nutrientes, río abajo.

Tal y como ya se ha comentado, la profundidad de procedencia del agua embalsada y su forma de incorporación al cauce (en salto con caída libre, en conducción a presión), es la clave de la calidad del agua río abajo y por tanto son también aspectos a tener en consideración. En el caso de aprovechamientos hidroeléctricos, donde la conducción forzada (a presión) del agua hasta las turbinas es obligada, hay la opción de instalar turbinas con un diseño que favorece la aireación del agua y/o equipos de aireación propiamente (ASCE/EPRI, 1989). Con la primera opción, se consiguen resultados francamente significativos en cuanto a recuperación del contenido de oxígeno disuelto.

\section{Corrección de la eutrofia}

Si la eficacia de las medidas preventivas se fundamentaba en la precisión de las predicciones sobre la vocación trófica y su reflejo en fases 
tempranas del proyecto constructivo del nuevo embalse, del éxito de la prevención dependerá la necesidad o no de llevar a cabo medidas correctoras durante la explotación que, en cualquier caso pueden evaluarse como indispensables para embalses ya eutróficos.

La corrección de la eutrofia en embalses puede abordarse desde dos perspectivas no necesariamente excluyentes, pero sí de muy distinto alcance y ámbito de aplicación. Tal y como se recoge en la figura 1, una opción es la gestión y la otra el tratamiento.

\section{Gestión}

La gestión se refiere a actuaciones de manejo del agua embalsada y/o de las comunidades naturales acuáticas. Como muestra la tabla 3 , estas actuaciones son en buena medida, comunes a las de tipo preventivo expuestas en la tabla 2 , pero en este caso no se aplican para que no aparezcan los síntomas de la eutrofia sino para atenuar su manifestación o para que esta tenga lugar dentro de unos límites admisibles.

Como ya se ha indicado en el apartado sobre medidas preventivas, la reducción de los aportes de nutrientes en origen, bien sea mediante actuaciones directas (depuración de aguas residuales) o indirectas (restauración hidrológico-forestal de la cuenca y control de aportes difusos a partir de prácticas agropecuarias inadecuadas), debe ser siempre la opción de partida a considerar.

La conservación, o si es el caso la restauración hidrológico-forestal de la cuenca, además de evitar problemas de erosión y aterramiento

Tabla 3. Principales opciones para la corrección de la eutrofia en embalses y/o la atenuación de sus síntomas. Main options for eutrophy correction in reservoirs and/or for mitigation of its'symptoms.

\begin{tabular}{|c|c|c|}
\hline $\begin{array}{l}\text { TIPO DE } \\
\text { ACTUACIÓN }\end{array}$ & $\begin{array}{l}\text { NIVELES DE } \\
\text { APLICACIÓN }\end{array}$ & $\begin{array}{l}\text { POSIBLES ASPECTOS A CONSIDERAR } \\
\text { O SOBRE LOS QUE ACTUAR }\end{array}$ \\
\hline Gestión & Cuenca tributaria & $\begin{array}{l}\text { - Depuración de aguas residuales } \\
\text { - Desviación ("by-pass") de tributarios } \\
\text { - Control de la contaminación difusa } \\
\text { - Restauración hidrológico-forestal }\end{array}$ \\
\hline \multirow[t]{3}{*}{ Gestión } & Agua embalsada & $\begin{array}{l}\text { - Curva-guía de explotación del nivel de embalse y control } \\
\text { de tiempo de residencia medio global y selectivo } \\
\text { - Programación de vaciados periódicos } \\
\text { - Instalación de equipos de aireación hipolimnética o de mezcla } \\
\text { vertical de la columna de agua }\end{array}$ \\
\hline & $\begin{array}{l}\text { Comunidades Acuáticas } \\
\text { del embalse }\end{array}$ & $\begin{array}{l}\text { - Modificación de la estructura trófica } \\
\text { - Manejo de zonas de cola de embalses como "filtros verdes" }\end{array}$ \\
\hline & $\begin{array}{l}\text { Agua y comunidades } \\
\text { río abajo }\end{array}$ & $\begin{array}{l}\text { - Aireación de las aguas salientes del embalse } \\
\text { - Aumento de la capacidad de aireación del cauce } \\
\text { - Implantación de un régimen de caudales de mantenimiento }\end{array}$ \\
\hline \multirow[t]{3}{*}{ Tratamiento } & Agua embalsada & $\begin{array}{l}\text { - Floculantes y precipitantes } \\
\text { - Oxidantes }\end{array}$ \\
\hline & Sedimentos del embalse & $\begin{array}{l}\text { - Secado y aireación mecánica } \\
\text { - Extracción } \\
\text { • Oxidación }\end{array}$ \\
\hline & $\begin{array}{l}\text { Comunidades acuáticas } \\
\text { del embalse }\end{array}$ & $\begin{array}{l}\text { - Extracción mediante medios mecánicos } \\
\text { - Alguicidas y desinfectantes } \\
\text { - Aplicación de productos químico-biológicos }\end{array}$ \\
\hline
\end{tabular}


de embalses (que a su vez son un agravante de la eutrofización), puede contribuir eficazmente a la retención de nutrientes y a la conservación de los acumulados en el suelo.

El volumen de agua embalsada a lo largo del año, es un factor determinante en el estado trófico de los embalses (Pérez-Martinez et al., 1989) por lo que cabe plantearse la posibilidad de establecer una curva-guía de explotación del nivel de embalse, coordinada con una extracción selectiva del agua a distintas profundidades, según la época del año, de modo que se limite al máximo el desarrollo algal y la desoxigenación hipolimnética (Vicente et al., 2000). Esta curva-guía podría incluir vaciados periódicos del embalse (por ejemplo decenales), que además de mejorar la aireación del sedimento, permitirían actuaciones mecánicas sobre él, si fueran necesarias. El vaciado de embalses es, por otro lado, una forma de supervisar el estado de conservación de la presa y sus órganos de regulación, lo cual es obligatorio en Francia cada 10 años.

Desde hace años existen equipos y experiencia práctica en la inyección controlada de aire, oxígeno o agua en el hipolimnion de lagos y embalses (Jaeger, 1989; ASCE/EPRI, 1989). Estas técnicas, si bien permiten contener uno de los principales problemas de la eutrofización (el déficit de oxígeno), son bastante costosas, complejas (Ashley \& Hall, 1989) y pueden llegar a tener un efecto contrario al buscado en función de la escala de tiempo considerada para evaluar los resultados, por ejemplo si se resuspenden sedimentos (y materia orgánica) y se rompe la estratificación térmica de la columna de agua, alimentando la zona fótica con más nutrientes.

Por el contrario, en sistemas poco profundos y bajo determinadas condiciones y objetivos, la desestratificación (mezcla vertical forzada) de la columna de agua, puede ser una buena opción para evitar o reducir la manifestación del déficit de oxígeno disuelto de fondo, asociable a la eutrofización.

A nivel de comunidades acuáticas, se han realizado experiencias sobre modificación de la estructura trófica para limitar el desarrollo algal, fundamentalmente mediante la eliminación (Faafeng \& Brabrand, 1989; Reinersten et al., 1997) o la introducción de especies de peces filtradores (Proulx et al., 1993; Bertolo et al., 2000) o comedores de macrófitos. En todos los casos, los resultados conducen a una significativa correlación positiva entre la biomasa de peces filtradores y la de fitoplancton, sin duda debida al cambio de presión de predación sobre el zooplancton y su mayor eficiencia en el control del fitoplancton. En zonas como la península ibérica, que representan un "nicho ecológico" vacío para este tipo de especies por la falta de lagos, la introducción de especies de peces filtradores para el control de la eutrofia, como en algunas ocasiones se ha insinuado, podría conducir a resultados espectacularmente contrarios a los esperados.

En cualquier caso, la retirada de materia orgánica (vegetación acuática, peces) está claro que es una forma de reducir la carga de nutrientes que moviliza el ecosistema, de modo que debe considerarse como una opción de control de la eutrofia. En este sentido, la gestión (retirada) de la materia orgánica producida en zonas de cola de los embalses que funcionen como humedales (alta productividad) puede inscribirse como una medida de control de la eutrofia. Paradójicamente, suele ocurrir que este tipo de ambientes artificiales, dada la diversidad de especies que albergan, en especial de aves, son objeto de algún tipo de protección que limita las posibilidades de uso como "filtro verde" para el embalse.

Aún dentro de las medidas de gestión, en el río aguas abajo, las actuaciones de corrección de la eutrofia se centran en lo ya indicado para su prevención; es decir en maximizar la capacidad de oxigenación de las aguas, tanto a la salida del embalse como a lo largo del río, conservando como aspecto clave, un régimen de caudales de mantenimiento adecuado y un buen estado de la vegetación de ribera.

\section{Tratamiento}

En cuanto a la aplicación de tratamientos correctivos de la eutrofia, la tabla 3 recoge las 
principales opciones existentes. Cabe indicar que se trata de actuaciones en su mayoría inaplicables a grandes embalses o en general masas de agua importantes, tanto por su coste económico, como por su eficacia y posibilidades de control. Son medidas por tanto adecuadas para pequeñas masas de agua, de usos muy específicos y fácilmente controlables a todo nivel, como es el caso de balsas de riego, depósitos de abastecimiento de agua, lagunas para actividades recreativas, etc.

A nivel del agua, existe la opción de aplicar tratamientos con productos químicos floculantes, precipitantes y oxidantes, que reduzcan la carga orgánica en la columna de agua a favor de la formación de sedimento más o menos mineralizado, que después se podrá retirar o tratar.

Dentro de los tratamientos del sedimento, está su oxidación mediante productos químicos, su secado y aireación mecánica para su mineralización, todo ello combinado o no con su retirada (Sebetich \& Ferriero, 1997). Algunas experiencias de secado y aireación mecánica o retirada de sedimentos, realizadas sobre lagunas eutróficas de cierta extensión, pero poco profundas, se apuntan como soluciones eficaces en la reducción de la carga de sedimentos disponibles (Phillips \& Jackson, 1989).

En cuanto a tratamientos sobre comunidades de organismos acuáticos, está la aplicación de alguicidas y productos desinfectantes, con las limitaciones inherentes al manejo de este tipo de sustancias, en ecosistemas acuáticos abiertos. Está también la ya citada retirada mecánica de organismos acuáticos (vegetación, peces), como una opción de reducción de la carga de materia orgánica (y nutrientes) procesable por el embalse.

También se han realizado algunas experiencias de corrección de la eutrofia en pequeñas masas de agua, basadas en aplicar compuestos químico-biológicos, de acción múltiple, formados por coprecipitantes, floculantes y microorganismos nitrificantes con sus capacidades enzimáticas aumentadas mediante ingeniería genética. Se trata probablemente del campo por el que avanzará (o debería avanzar) el tra- tamiento de la eutrofia en pequeñas masas de agua, dado que se fundamente en la potenciación de reacciones y procesos naturales del propio ecosistema acuático.

\section{CONCLUSIONES}

La corrección definitiva del proceso de eutrofización pasa forzosamente por la eliminación, o al menos la atenuación de la fuente causante de la alteración: el aporte excesivo de nutrientes. El problema es que esta opción no es siempre posible y en cualquier caso suele ser de difícil ejecución, no sólo por el carácter difuso que pueden tener parte de las aportaciones, sino porque la retirada de nutrientes requiere tratamientos poco extendidos en los planes de saneamiento de cuencas. De esta forma, el control de la eutrofia tiende a centrarse habitualmente en el control de sus efectos o síntomas, más que en la erradicación de sus causas.

En el caso de embalses además, se trata de ecosistemas "condenados" a padecer un cierto grado de eutrofia al menos en su primer llenado, como consecuencia de la mineralización bajo el agua, de la materia orgánica presente en su vaso. Sólo por esta razón, y al margen del interés social y económico que tiene, resulta obligada la consideración de la eutrofia como un aspecto relevante más del proyecto técnico de cualquier nuevo embalse.

Para embalses nuevos que se juzguen inapelablemente necesarios, a partir del reconocimiento del lugar donde se proyectan y de las características técnicas de su presa y su cubeta, recogidas a nivel de anteproyecto, es posible mediante modelos sencillos, establecer la vocación trófica de la futura masa de agua artificial (Fig. 2). En función de los resultados de esta predicción, se debería tratar la eutrofización potencial como un condicionante económicamente relevante para embalses con determinados usos y objetivos, recogiendo si es el caso, dentro del propio proyecto técnico definitivo $\mathrm{y} / \mathrm{o}$ en el preceptivo estudio de efectos ambientales que lo acompaña, aquellas medidas pre- 

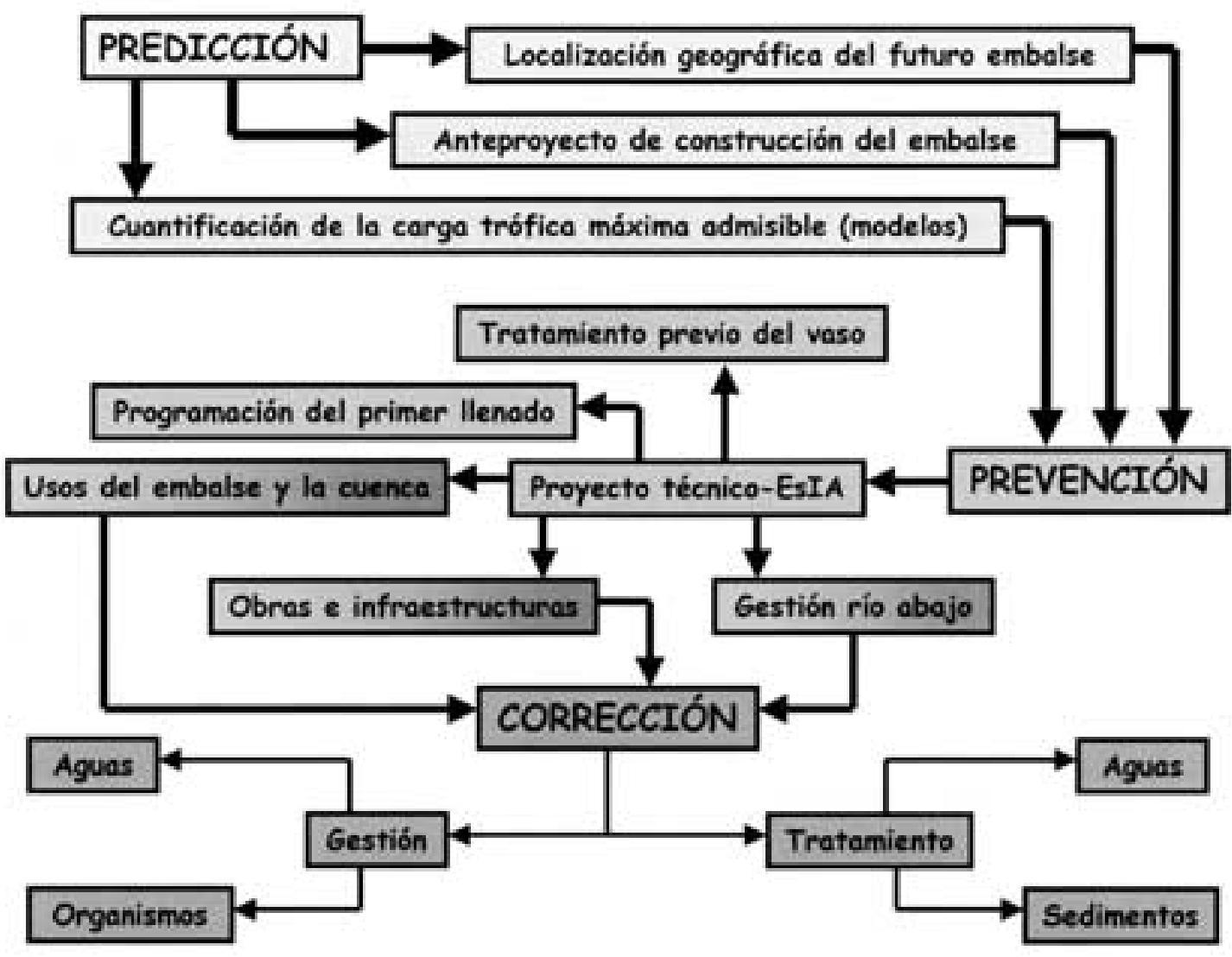

Figura 2. Principales medidas de control y gestión de la eutrofia a partir de la predicción del estado trófico de un anteproyecto de embalse, en la prevención a nivel de proyecto y en la corrección en un embalse en explotación (EsIA: Estudio de Impacto Ambiental). Main measures for eutrophication control and management, from the trophic state prediction in the reservoir's preliminary draft phase, the incorporation of preventive measures on the definitive draft phase, and the corrective actions when the reservoir is operative.

ventivas de la eutrofia que se consideren más adecuadas y que como mínimo podrán centrarse en el tratamiento previo del futuro vaso inundable del embalse, la programación del primer llenado y de los usos futuros del embalse, el diseño de elementos estructurales y las actuaciones y medidas de gestión necesarias para el tramo de río que quedará regulado.

Si a pesar de todo la eutrofia se manifiesta por encima de lo deseable, o se está delante de un embalse eutrófico ya en explotación, pueden aplicarse medidas correctoras a distintos niveles (aguas, sedimentos, organismos), recogidas en forma de actuaciones de gestión, bastante coin- cidentes con las posibles actuaciones preventivas, o bien como tratamientos, en ese caso fundamentalmente adecuados para pequeños volúmenes de agua.

La disponibilidad de tomas de agua a diferentes niveles de embalse y la gestión selectiva de los tiempos de residencia del agua, se presentan como medidas eficaces en el control de la eutrofia, con una capacidad a la vez preventiva y correctora.

Desde la fase de proyecto a la de explotación de un embalse, las opciones de control de la eutrofia recogidas de forma genérica en la figura 2 , tienden a ser cada vez menos eficien- 
tes, bien sea por su mayor coste o la creciente disminución de la capacidad de autorecuperación del embalse potencialmente eutrófico. Puede ocurrir perfectamente que no se puedan controlar del todo los efectos de la eutrofia, a pesar de las medidas tomadas y de las inversiones realizadas, dado que otros factores no controlables (meteorología, aportación hidrológica, etc.), poco previsibles (situaciones accidentales, etc.) o poco controlables (vertidos ilegales, etc.), pueden modificar de forma significativa el sentido de los resultados, al menos transitoriamente.

Por último, la eutrofización de embalses no hay que interpretarla como una perturbación localizada sólo aguas arriba de las presas, sino también con consecuencias importantes río abajo, donde por las propias características del ecosistema acuático lótico, el control de la eutrofia admite menos opciones, pero las disponibles son más simples y eficaces, todas ellas centradas básicamente en fomentar la aireación y evitar o promover (según los objetivos) zonas de acumulación (alto tiempo de residencia) de nutrientes y materia orgánica.

\section{BIBLIOGRAFÍA}

ASCE/EPRI. 1989. Civil Engineering Guidelines for Planning and Designing Hydroelectric Developments. American Society of Civil Engineers (ASCE) y Electric Power Research Institute (EPRI). ASCE/EPRI Guides 1989, Vol. 1. N.Y. USA.

ASHLEY, K. I. \& K. J. HALL. 1989. Factors influencing oxygen transfer in hypolimnetic aeration systems. Verh. Internat. Verein. Limnol., 24: 179-183.

BERTOLO, A., F. LESCHER-MOUTOUÉ \& G. LACROIX. 2000. Interaction effects of depth and planktivorous fish on plankton biomass. Verh. Internat. Verein. Limnol., 27: 1747-1751.

CASSIDY, R. A. 1989. Water temperature, dissolved oxygen and turbidity control in reservoir releases. In: Alternatives in regulated river management. J.A. Gore \& G.E. Petts (eds.): 28-62. CRC Press. Boca Raton, Florida (USA).
FAAFENG, B. A. \& A. BRABRAND. 1989. Biomanipulation of a small, urban lake-removal fish exclude bluegreen blooms. Verh. Internat. Verein. Limnol., 24: 597-602.

JAEGER, D. 1989. TIBEAN- a new hypolimnetic aeration plant. Verh. Internat. Verein. Limnol., 24: 184-187.

LEE, G. F., W. RAST, R. A. JONES \& J. L. ORTIZ. 1980. Progresos recientes en la estimación de la respuesta de los lagos y embalses a las aportaciones de nutrientes. Centro de Estudios y Experimentación del Centro de Estudios Hidrográficos (MOPU). Publicación $n^{\circ} 137$. Madrid

MARGALEF, R. 1976. Biología de los embalses. Investigación y Ciencia, 1: 50-62.

MOSS, B., J. STANSFIELD \& K. IRVINE. 1989. Problems in the restoration of a hypereutrophic lake by diversion of a nutrient-rich inflow. Verh. Internat. Verein. Limnol., 24: 568-572.

PALAU, A. 1991. Evolution of annual chlorophyll concentration in a high mountain pump-storage reservoir. Verh. Internat. Verein. Limnol., 24: 1401-1404.

PALAU, A. 1994. Los mal llamados caudales "ecológicos". Bases para una propuesta de cálculo. OP (Obras Públicas), 28 (2): 84-95.

PÉREZ-MARTÍNEZ, C., R. MORALES-BAQUERO \& P. SÁNCHEZ-CASTILLO. 1989. The effect of the volume decreasing on the trophic status in four reservoirs from Southern Spain. Verh. Internat. Verein. Limnol., 24: 1382-1385.

PHILLIPS, G. \& R. JACKSON. 1989. The control of eutrophication in very shallow lakes, the Norfolk Broads. Verh. Internat. Verein. Limnol., 24: 573-575.

PROULX, M., F. R. PICK, A. MAZUMDER \& D. R. S. LEAN. 1993. Effects of plancktivorous fish on phytoplancton biomass and community structure. Verh. Internat. Verein. Limnol., 25: 331-334.

RAVERA, O. 1989. The effects of hypolimnetic oxygenation in the shallow and eutrophic Lake Comabbio (Northern Italy) studied by enclosure. Verh. Internat. Verein. Limnol., 24: 188-194.

REINERSTEN, H., J. I. KOKSVIK \& A. HAUG. 1997. Effects of fish elimination on the phytoplankton and zooplankton in a small eutrophic lake. Verh. Internat. Verein. Limnol., 26: 593-598. 
RYDING, S. O. \& W. RAST. 1993. Le contrôle de l'eutrophisation des lacs et des reservoirs. Ed. Masson. UNESCO. París.

SEBETICH, M. J. \& N. FERRIERO. 1997. Lake restoration by sediment dredging. Verh. Internat. Verein. Limnol., 26: 776-781.

STRASKRABA, M. 1999. Retention Time as a Key Variable of Reservoir Limnology. In: Theoretical Reservoir Ecology and its Applications. J.G. Tundisi \& M. Straskraba (eds.): 385-410. International Institute of Ecology. Brazilian Academy of Sciences and Backhuys Publishers.

TOJA, J. 1982. Control de la eutrofia en embalses por utilización selectiva de agua a distintas pro- fundidades. Obras Públicas (Abril-Mayo): 223231.

VICENTE, E., A. CAMACHO, M. D. SENDRA, D. SANCHIS, J. M. SORIA, M. J. DASÍ \& M. R. MIRACLE. 2000. Limnological management of the Amadorio Reservoir (Spain) during an extremely dry summer. Verh. Internat. Verein. Limnol., 27: 2298-2302.

WETZEL, R. G. 1981. Limnología. Ed. Omega. Barcelona.

WILSON, H. M. \& P. E. O'SULLIVAN. 1993. The control of eutrophication of small shallow lakes in the United Kingdom: The legal framework. Verh. Internat. Verein. Limnol., 25: 461-464. 